
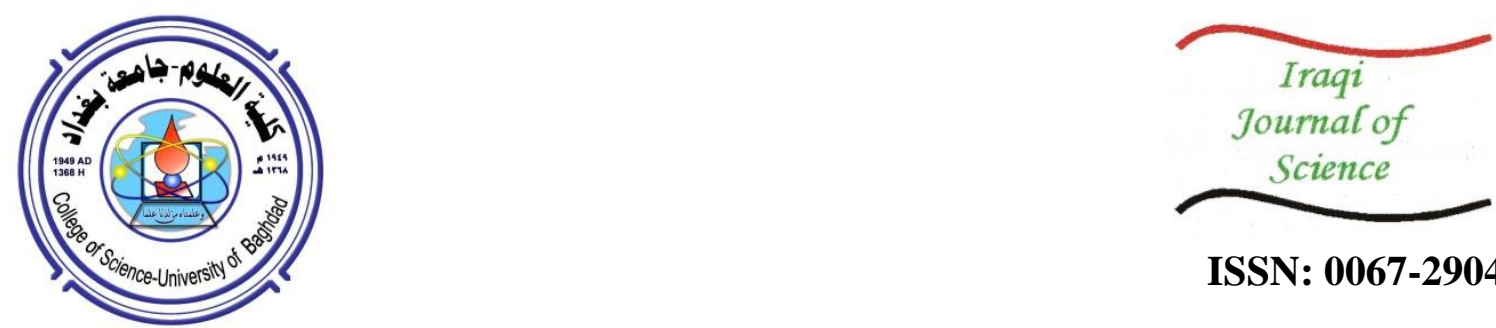

ISSN: 0067-2904

\title{
On Invariant Approximations in Modular Spaces
}

\section{Nadia Jasim Mohammed, Salwa Salman Abed*}

Department of Mathematic, College of Education for Pure Sciences, Ibn Al-Haitham, University of Baghdad, Baghdad, Iraq

Received: $10 / 11 / 2020$

Accepted: 16/3/2021

\begin{abstract}
This article is devoted to presenting results on invariant approximations over a non-star-shsped weakly compact subset of a complete modular space by introduced a new notion called S-star-shaped with center $f:$ if $S: B \rightarrow B$ be a mapping and $\forall e \in B, \lambda S e+(1-\lambda)(f-S f) \in B, f \in B$. Then the existence of common invariant best approximation is proved for Banach operator pair of mappings by combined the hypotheses with Opial's condition or demi-closeness condition
\end{abstract}

Keywords: Banach operator pair, best approximation, fixed point, modular space, $S_{\text {-non-expansive mappings. }}$

$$
\begin{aligned}
& \text { حول التقريب الثابت في فضاءات الوحدات } \\
& \text { نادية جاسم عحمد, سلوى سلمان عبد } \\
& \text { قسم الرياضيات ، كلية التربية للعلوم الصرفه ، ابن الهيثم ، جامعة بغداد ، بغداد، العراق } \\
& \text { الخلاصة } \\
& \text { كُرست هذه المقالة لتقديم نتائج حول التقريب الثابت تحت شرط مجموعة جزئية متراصة بضعف في فضاء } \\
& \text { الوحدات الكامل من خلال تقديم مفهوم جديد يسمى S-star-shaped مع المركزf : : إذا كان } \\
& \text { و } S: B \rightarrow B
\end{aligned}
$$

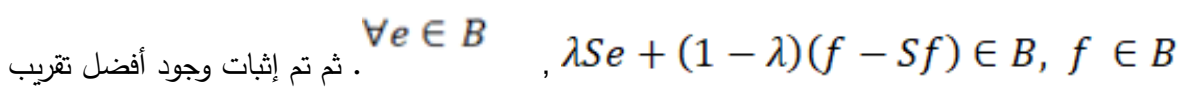

$$
\begin{aligned}
& \text { ثابت مشترك لتطبيقين من نوع زوج مؤثر بناخ وذلك من خلال دمج الفرضيات مع شرط أوبل أو شرط شبه } \\
& \text { القرب. }
\end{aligned}
$$

\section{Introduction and Preliminaries}

Recent paper contains applications of fixed point of non-expansive mappings in a modular space which is known as the following:

Definition 1.1 [1] Let ${ }^{\mathrm{B}}$ be real linear space over $R$, a function $\gamma: \mathrm{B} \rightarrow(0, \infty)$ is called modular if for $e, h \in B$ and $\alpha, \beta \in R$.

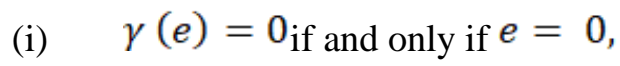

(ii) $\quad \gamma(\alpha e)=\gamma(e)$ with $|\alpha|=1$,

*Email: salwaalbundi@yahoo.com 
(iii) $\quad \gamma(\alpha e+\beta h) \leq \gamma(e)+\gamma(h)$ if and only if, $\alpha, \beta \geq 0$.

If (iii) replaced by (iii) ' $\gamma(\alpha e+\beta h) \leq \alpha \gamma(e)_{+} \beta \gamma(h)$, for $\alpha, \beta \geq 0, \alpha+\beta=1$, for all $e, h$

$\in \mathrm{B}$, then $\gamma$ is called convex modular.

Definition 1.2 [1] If $\gamma$ is a convex modular on $\mathbf{B}$. A corresponding set $\mathbf{B}_{\gamma}$ $=\{e \in \mathrm{B}: \gamma(\alpha e) \rightarrow 0$ whenever $\alpha \rightarrow 0\}$ is called convex modular space.

The distance between two vectors $e, h_{\text {is }} d_{\gamma}(e, h)=\gamma(e-h)$. The collection of all balls in a modular space $\mathbf{B}_{Y}$ generates a locally convex Hausdorff topological linear space [2]. Many works in this space can be found in [3-6]. Recently, Abed and Abdul Jabbar [7-10] introduced the concept of normalized duality mappings in the real convex modular spaces proved some of its properties which related to uniformly smooth of these spaces. Also, they presented some results concerning the convergence and equivalence iterative sequences for multivalued strongly pseudo-contractions mappings. Now, we recall the concepts required for our results. Let ${ }^{B}$ be a convex real modular space with dual ${ }^{\mathrm{B}_{Y}^{\prime}}$ (for details of dual ${ }^{\mathrm{B}_{Y}^{\prime}}$, see [8])

Definition 1.3 [8]

(1) A sequence $\left\{e_{n}\right\} \subset \mathrm{B}_{\gamma}$ is said to be

(a) $\quad \gamma$-convergent to $e \in \mathrm{B}_{\gamma}$ and write $e_{n} \stackrel{\gamma}{\rightarrow} e$ if $\gamma\left(e_{n}-e\right) \rightarrow 0$ as $\mathrm{n} \rightarrow \infty$.

(b) $\quad \gamma_{-}$Cauchy whenever $\gamma_{(}\left(e_{n}-e_{m}\right) \rightarrow 0$ as $n, m \rightarrow \infty$.

(2) If any $\gamma_{-}$Cauchy sequence in ${ }^{B_{\gamma}}$ is $\gamma_{-}$convergent then ${ }^{B_{\gamma}}$ is called $\gamma_{-}$complete.

(3) If for any sequence $\left\{e_{n}\right\} \subset \subset^{B \subset B_{\gamma}}$ is $\gamma_{-}$convergent to a point in $B$ then $B$ is called $\gamma_{-}$closed. And, if any sequence $\left\{e_{n}\right\} \subset^{B} \subset \mathrm{B}_{\gamma}$ has a $\gamma_{-}$convergent subsequence then $B_{\text {is }}$ called $\gamma_{-}$ compact.

Definition (1.4) [7] A sequence $\left\{e_{n}\right\}$ in ${ }^{B_{Y}}$ is said to be weakly convergent if there is an $e \in{ }^{B_{Y}}$ such that for every $P \in \mathrm{B}_{Y}^{\prime}, \lim _{n \rightarrow \infty} \gamma\left(P e_{n}-P e\right)=0$ This denoted by $e_{n} \stackrel{\mathrm{w}}{\rightarrow} e$.

Definition (1.5) [3] Let $B$ be a subset of a convex real modular space ${ }^{B}$. For $e_{0} \in B$. $B A\left(e_{0}\right)$ denotes the set of best B-approximation to $x_{0}$,

$B A\left(e_{0}\right)=\left\{h \in B: \gamma\left(h-e_{0}\right)=d\left(e_{0}, B\right)\right\}$

where $d\left(e_{0}, B\right)=\inf f_{z \in B} \gamma\left(z-e_{0}\right)$

Definition (1.6) [3] Let $S, T: B \rightarrow B$, then $F(T)$ denotes the set of all fixed points of $T$ and $F(S, T)$ is the set of all common fixed points of $S$ and $\mathrm{T}$.

Definition (1.7) [9] A mapping $S$ is called T- $\gamma$-contraction if for all $e, f \in B, \gamma(S e-S f) \leq \alpha \gamma(T e-T f)$,

$\alpha \in(0,1)$. And it is called T- $\gamma_{\text {-non-expansive if for all }} e, f \in B, \gamma(S e-S f) \leq \gamma(T e-T f)$.

Definition (1.8) [7] A modular space ${ }^{B}$ satisfies Opial's condition if for every sequence $\left\{e_{n}\right\} \subseteq B$ weakly convergent to $e \in \mathrm{B}, \lim _{n \rightarrow \infty} \inf \gamma\left(e_{n}-e\right)<\lim _{n \rightarrow \infty} \inf \gamma\left(e_{n}-h\right)$ holds, for all $h \neq e$.

Definition (1.9) [7] Let $B \subseteq \mathrm{B}, a$ mapping $T: B \rightarrow \mathrm{B}$ is called demi-closed on $B$. If for every sequence $\left\{e_{n}\right\} \subseteq B$, $\left\{e_{n}\right\}$ converges weakly to $e \in B$ and $\left\{T e_{n}\right\}_{\text {converges strongly to }} h \in \mathrm{B}$, implies $h=T e$.

Definition (1.10) [2] Let $B \subseteq \mathrm{B}, B$ is called

i) Convex if $\lambda e+(1-\lambda) f \in B, \forall e, f \in B, \forall \lambda \in[0,1]$

ii) Star-shaped with center $f \in B$ if $\lambda e+(1-\lambda) f \in B, \forall e \in B$.

Main Results

In the following, a simple modification of star-shaped: 
Definition (2.1) Let $\emptyset \neq B \subset B$ and $S: B \rightarrow B$ be a mapping, $B$ is called S-star-shaped with center $f$ if $\forall e \in B, \lambda S e+(1-\lambda)(f-S f) \in B$.

Theorem (2.2) Let $\mathrm{B}$ be a complete space $e_{\circ} \in \mathrm{B}$ and $S: \mathrm{B} \rightarrow \mathrm{B} \ni S(\partial B) \subseteq B$

If $B$ is w-compact and $S_{\text {-star-shaped such that }}$

i) $\quad S$ is $\gamma$-non-expansive on $B A\left(e_{\circ}\right)$.

ii) $\gamma\left(\left(S e-S e_{\circ}\right) \leq \gamma_{(} e-e_{0}\right), \forall e \in B A\left(e_{\circ}\right)$.

iii) $I-S$ is $\gamma$-demiclosed on $B A\left(e_{\circ}\right)$.

Then $\mathrm{S}$ has a fixed point closed set to $e_{\mathrm{o}}$.

Proof: Firstly, we prove $\mathrm{S}$ is self-mapping on $B A\left(e_{0}\right)$. Let $e \in B A\left(e_{0}\right)$, then $\gamma\left(S e-e_{0}\right) \leq \gamma\left(S e-S e_{0}\right) \leq \gamma\left(e-e_{0}\right)$.

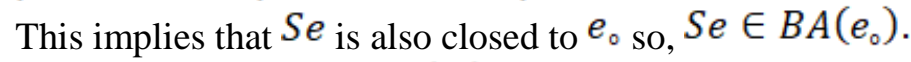

Let $f$ be star center of $B A\left(e_{0}\right)$.

Define $S_{m_{i}} e=m_{i} S e+(1-m i)(f+S f)$

Where $\left\{m_{i}\right\}$ real sequence, $0<m i<1$ and $\lim _{i \rightarrow \infty} m_{i}=1, e \in B A\left(e_{\circ}\right)$.

To prove $S_{n}$ is $\gamma$-contraction, follow:

$$
\text { let } \begin{aligned}
e, g \in B A\left(e_{\circ}\right), \gamma\left(S_{n} e-S_{n} g\right) & =\gamma\left(m_{i} S_{n} e-m_{i} S_{n} g\right) \\
& =m_{i} \gamma\left(S_{n} e-S_{n} g\right) \\
& \leq m_{i} \gamma(e-g)
\end{aligned}
$$

since $m_{i}<1$ then $S_{n}$ is $\gamma_{\text {-contraction. }}$

By argument in [11] $\forall n, S_{n}$ has a fixed point $e_{n}$ is $B A\left(e_{\circ}\right), i . e, S_{n} e_{n}=e_{n}, \forall n$

Since B is w-compact, then the sequence of fixed point $\left\{e_{n}\right\}$ has a convergent subsequence $\left\{e_{n_{i}}\right\} \mathrm{w}-$ convergent to $\bar{e}$, say. Now,

$$
\begin{aligned}
\gamma\left(e_{n_{i}}-S e_{n_{i}}\right) & =\gamma\left(S_{n} e_{n_{i}}-S e_{n_{i}}\right) \\
& =\gamma\left(m_{n_{i}} S e_{n_{i}}+\left(1-m_{n_{i}}\right)(f+S f)-S e_{n_{i}}\right. \\
& =\left(m_{n_{i}}-1\right) S e_{n_{i}}+\left(1-m_{n_{i}}\right)(f+S f) \\
& \rightarrow 0 \quad \text { as } i \rightarrow \infty .
\end{aligned}
$$

Since $I-S$ is demi-closed, then, $0=(I-S) \bar{e}$ and $\bar{e}=S \bar{e}$, thus $S$ has a fixed point $\bar{e}_{\text {closest to }} e_{\text {o }}$.

Now, we recall the definition of Banach operator pairs, for a mapping $T: \mathrm{B} \rightarrow \mathrm{B}, \quad F(T):=\{e \in \mathrm{B}: T e=e\}$

Definition (2.3) [4] The ordered pair $(S, T)$ of two self-mappings $S$ and $T$ of $\mathbf{B}$ is called a Banach operator if $S(F(T) \subseteq F(T)$ i.e. the set $F(S)$ is T-invariant.

Remark (2.4) If $S$ and $T$ are commute on $B$ then $(S, T)$ must be a Banach operator. But the converse is not true. For example:

Example (2.5) Consider $\mathrm{B}=R$ and

$S(e, f)=\left(e^{2}+f^{2}+e-1, e^{2}+f^{2}+t-1\right)$

$T(e, f)=\left((e-f)^{2}+2 e-f,(e-f)^{2}+e\right)$

For any $(e, f) \in \mathrm{B}$. Then

$F(S)=\left\{(e, f) \in B: e^{2}+f^{2}-1=0\right\}$

$F(T)==\{(e, f) \in \mathrm{B}: s-t=0$ or $s-t+1=0\}$

So, (i) $S(F(T)) \subseteq F(T) \rightarrow(S, T)$ is Banach operator pair on $B$.

(ii) $(T, S)$ is not Banach operator pair, since for $(1,0) \in F(S), T(1,0)=(3,2) \notin F(S)$.

Remark (2. 6) [4] In general metric spaces the pair $(S, T)$ is Banach operator $\Leftrightarrow S$ and $T$ commute on $F(T)$. In the above Example (2.5-i) $T, S$ are commute on the set $F(T)$, so, $(S, T)$ is Banach operator by Remark (2.4). 
To prove a common fixed point theorem, we need the following Lemma in general metric spaces.

Lemma (2. 7) [12] Let $B$ be a closed subset of a metric space $(B, d)$ and $S, T: B \rightarrow B$. If $(S, T)$ is a Banach operator pair and $S$ is T- $\gamma$-contraction on $B, T$ is continuous, $F(T) \neq \emptyset \overline{S(B)}$ is complete. Then $F(S, T)=$ singleton.

Theorem (2.8) Let $B$ be a w-compact subset of convex real modular space ${ }^{\text {B }, ~} B$ is S-star-shaped respect to $f \in B$. Let $(S, T)$ be a Banach operator on B $f \in F(T)$. If $T$ is both weakly continuous and strongly continuous on $\mathrm{B}, F(T)$ is star-shaped with $f, \overline{S(B)}$ is complete and if either

(i) B satisfies Opial's condition.

or

(ii) $I-S$ is demiclosed on $\mathrm{B}$.

Then $F(S, T) \neq \emptyset$

Proof: Let $\left\{m_{i}\right\}$ be real sequence $\exists 0<m_{i}<1, m_{i} \rightarrow 1$ as $i \rightarrow \infty$. Define

$S_{i}(e)=m_{i} S e+\left(1-m_{i}\right)(f+S f), \forall e \in B$

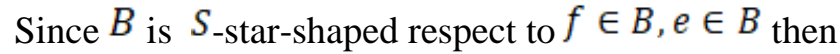

- $\forall i, S_{i}: B \rightarrow B$, since B is S-star-shaped with $f$.

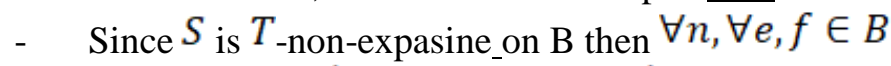

$$
\begin{aligned}
\gamma\left(S_{i} e-S_{i} f\right) & =\gamma\left(m_{i} S(e)-m_{i} S(f)\right) \\
& \leq m_{i} \gamma(T(e)-T(f))
\end{aligned}
$$

$\rightarrow$ each $S_{i}$ is g- $\gamma$-contraction on $B$.

- For each $i,\left(S_{i}, T\right)$ is a Banach operator pair on $B$, since $(\mathrm{S}, \mathrm{T})$ is a Banach operator pair on $B$, since $(S, T)$ is a Banach operator, for $e \in F(T) \rightarrow S(e) \in F(T)$.

$\rightarrow S_{i}(e)=m_{i} S e+\left(1-m_{i}\right)(f+S f) \in F(T)$

By the fact $F(T)$ is S-star-shaped with $f \in F(T)$

- The completeness of $\overline{S(B)} \rightarrow$ completeness of $\overline{S_{1}}(B)$. And w-compactness of $B \rightarrow B$ is closed.

Next, by Lemma (2.7), $\forall i, \exists e_{i} \in B \ni e_{i} \in F\left(S_{i}, T\right)$

Since w-compactness of $B$ and w-continuity of $T$ implies w-compactness of $F(T) \subseteq B, \exists\left\{e_{i_{k}}\right\}$ subsequence converges weakly to $e_{0} \in F(T)$.

Now, we must prove $e_{\circ} \in F(S, T)$.

As known w-compactness weak bounded and thus bounded $\rightarrow\left\{S e_{i_{k}}\right\} \subseteq B$ is bounded.

Since

$$
\begin{aligned}
& \gamma\left((S-T) e_{i_{k}}\right)=\gamma\left(\left(m_{i}-1\right) S e_{n_{i}}+\left(1-m_{i}\right)(f+S f)\right) \\
&= \gamma\left(\left(1-m_{i}\right)\left(f+S f-S e_{n_{i}}\right)\right) \\
& \gamma\left((T-S) e_{n_{i}}\right) \leq\left(m_{i}-1\right)\left(\gamma(f+S f)+\gamma\left(S e_{n_{i}}\right)\right) \\
& \rightarrow 0 \text { as } i \rightarrow \infty
\end{aligned}
$$

Now, if the hypothesis (i) holds and $T e_{\circ} \neq S e_{\circ}$.

Form (**) and T-contraction and T-non-expansive, we get

$$
\begin{aligned}
\lim _{k \rightarrow \infty} \inf \gamma\left(T e_{i_{k}}-T e_{\circ}\right) & <\lim _{k \rightarrow \infty} \inf \gamma\left(T e_{i_{k}}-S e_{\circ}\right) \\
& \leq \lim _{k \rightarrow \infty} \inf \gamma\left((T-S) e_{i_{k}}\right)+\lim _{k \rightarrow \infty} \inf \left(S e_{n_{i_{k}}}-S e_{\circ}\right) \\
& \leq \lim _{k \rightarrow \infty} \inf \left(T e_{i_{k}}-T e_{\circ}\right)
\end{aligned}
$$

which is a contraction. $S_{0}, T e_{0}=S e_{0}$ and then $e_{\circ} \in F(S, T)$.

If the hypothesis (ii) holds, i.e, if $T-S$ is demiclosed on $B$. Then by (**) and w-convergence of $\left\{e_{i_{k}}\right\}$ to $e_{0} \rightarrow(T-S) e_{0}=0$ 
i.e. $T e_{0}=S e_{0} \rightarrow e_{0} \in F(S, T)$.

As application of theorem (2.8) to best approximation, we present the following:

Theorem (2.9) Assume that B satisfies Opial's condition, $S, T: B \rightarrow B$ and $B \subseteq B$ with $S(\partial B \cap B) \subseteq B$ and $\overline{S(B)}$ complete. Let $e_{\circ} \in F(S, T) \exists \emptyset=B A\left(e_{\circ}\right)$ is w-compact and S-starshaped w.r.t. $f \in F(T)$. If $(S, T)$ is Banach operator pair on $B A\left(e_{\circ}\right), S$ is $T$-non-expansive on $B A\left(e_{\mathrm{o}}\right) \cup\left\{e_{\mathrm{o}}\right\}$, and if $T$ is both weakly and strongly continuous on $B A\left(e_{\mathrm{o}}\right) . F(T)$ is $S-$ starshaped w.r.t. $f$ and $T\left(B A\left(e_{0}\right)\right) \subseteq B A\left(e_{0}\right)$ then $B A\left(e_{0}\right) \cap F(S, T) \neq \emptyset$.

\section{Proof:}

If $e_{0} \in B$ then $e_{0} \in B A\left(e_{0}\right) \cap F(S, T)$ and the result holds.

Now, if $e_{0} \notin B \rightarrow B A\left(e_{0}\right) \subseteq \partial B \cap B \rightarrow S: B A\left(e_{0}\right) \rightarrow C$.Since $S\left(e_{0}\right)=T\left(e_{0}\right)=e_{0}$ and $S$ is Tnon-expasive. On $B A\left(e_{0}\right) \cup\left\{e_{0}\right\} \rightarrow \forall h \in B A\left(e_{0}\right)$,

$\gamma\left(S h-e_{\mathrm{o}}\right)=\gamma\left(S h-S e_{\mathrm{o}}\right) \leq \gamma\left(T h-T e_{\mathrm{o}}\right)=d\left(e_{\mathrm{o}}, B\right)$.

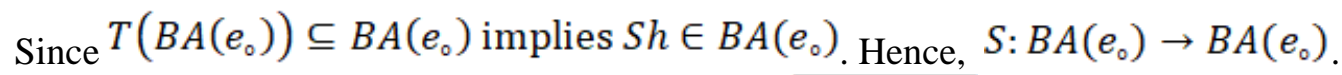

The completeness of $\overline{S(B)} \rightarrow$ completeness of $\overline{S\left(B A\left(e_{0}\right)\right)}$ and $B A\left(e_{0}\right) \cap F(T)$ is $S$-star-shaped w.r.t. $f$. By applying Theorem (2.8) to $\mathrm{S}$ and $\mathrm{T}$ on $B A\left(e_{\circ}\right)$ we get $B A\left(e_{\circ}\right) \cap F(S, T) \neq \emptyset$

\section{References}

1. H. Nakano, 1950. "Modulared semi-ordered linear spaces", Tokyo Math. Book Ser., vol. 1, Maruzen Co., Tokyo.

2. S. S. Abed, 2017. "On invariant best approximation in modular spaces", Global Journal of Pure and Applied Mathematics, 13(9): 5227-5233.

3. S. S. Abed and N.J. Mohammed, 2018. "Best approximation in modular spaces by type of nonexpansive maps", Iraqi Journal of Science, 59(3B): 1444-1446.

4. N. Hussain, M, A. Khamsi, and A. Latif, A., 2011. "Banach operator pairs and common fixed points in modular function spaces”, Fixed Point Theory and Applications, 75: 1-12.

5. R. Chen and X. Wang, 2013. "Fixed point of nonlinear contractions in modular spaces", Journal of Inequalities and Applications, 399: 1-9.

6. M. Paknazar, M. Eshaghi, Y. Cho, and M. Vaezpour. 2013. "A Pata-type fixed point theorem in modular spaces with application", Fixed Point Theory and Applications, 239: 1-11.

7. S.S. Abed, and M.F. Abdul Jabbar. "Some Results on Normalized Duality Mappings and Approximating Fixed Points in Convex Real Modular Spaces", accepted in Baghdad journal of science.

8. M.F. Abdul Jabbar, and S. S. Abed, 2020. "The convergence of iteration scheme to fixed points in modular spaces" Iraqi Journal of Science, 60(10): 2197-2202.

9. S.S. Abed, and M.F. Abdul Jabbar, 2020. "Approximating fixed points in modular spaces", Karbala International Journal of Modern Science, 6(2): 121-128.

10. S.S. Abed, and M.F. Abdul Jabbar, 2020. "Equivalence between iterative schemes in modular spaces", Journal of Interdisciplinary Mathematics, 22(8): 1529-1535.

11. K. Kuaket, and P. Kumam, 2011. "Fixed points of asymptotic point-wise contractions in modular spaces", Appl. Math. Lett. 24: 1795-1798.

12. J.Chen and Z. Li, 2007. "Common Fixed Points for Banach Operator Pairs in Best Approximation”, Journal of Mathematical Analysis and Applications,.336: 1466-1475. 